\title{
The prevalence of dementia in a Portuguese community sample: a 10/66 Dementia Research Group study
}

\author{
Manuel Gonçalves-Pereira 1* 1 , Ana Cardoso', Ana Verdelho², Joaquim Alves da Silva ${ }^{1,3}$, \\ Manuel Caldas de Almeida ${ }^{4,1}$, Alexandra Fernandes ${ }^{5}$, Cátia Raminhos ${ }^{1}$, Cleusa P. Ferri ${ }^{6,7}$, A. Matthew Prina ${ }^{6}$, \\ Martin Prince ${ }^{6}$ and Miguel Xavier ${ }^{1}$
}

\begin{abstract}
Background: Dementia imposes a high burden of disease worldwide. Recent epidemiological studies in European community samples are scarce. In Portugal, community prevalence data is very limited. The 10/66 Dementia Research Group (DRG) population-based research programmes are focused in low and middle income countries, where the assessments proved to be culture and education fair. We applied the 10/66 DRG prevalence survey methodology in Portugal, where levels of illiteracy in older populations are still high.

Methods: A cross-sectional comprehensive one-phase survey was conducted of all residents aged 65 and over of two geographically defined catchment areas in Southern Portugal (one urban and one rural site). Nursing home residents were not included in the present study. Standardized 10/66 DRG assessments include a cognitive module, an informant interview and the Geriatric Mental State-AGECAT, providing data on dementia diagnosis and subtypes, mental disorders including depression, physical health, anthropometry, demographics, disability/functioning, health service utilization, care arrangements and caregiver strain.

Results: We interviewed 1405 old age participants (mean age 74.9, SD = 6.7 years; 55.5\% women) after 313 (18.2\%) refusals to participate.

The prevalence rate for dementia in community-dwellers was 9.23\% (95\% Cl 7.80-10.90) using the 10/66 DRG algorithm and 3.65\% (95\% Cl 2.97-4.97) using DSM-IV criteria. Pure Alzheimer's disease was the most prevalent dementia subtype (41.9\%). The prevalence of dementia was strongly age-dependent for both criteria, but there was no association with sex.

Conclusions: Dementia prevalence was higher than previously reported in Portugal. The discrepancy between prevalence according to the 10/66 DRG algorithm and the DSM-IV criteria is consistent with that observed in less developed countries; this suggests potential underestimation using the latter approach, although relative validity of these two approaches remains to be confirmed in the European context. We improved the evidence base to raise awareness and empower advocacy about dementia in Portugal, so that the complex needs of frail older people may be met in better ways.
\end{abstract}

Keywords: Dementia, Alzheimer's disease, Epidemiology, Prevalence, Questionnaires, Cross-cultural psychiatry

\footnotetext{
* Correspondence: gpereira@nms.unl.pt

${ }^{1}$ Chronic Diseases Research Center (CEDOC), Nova Medical School |

Faculdade de Ciências Médicas, Universidade Nova de Lisboa, Campo

Mártires da Pátria 130, 1169-056 Lisbon, Portugal

Full list of author information is available at the end of the article
}

(c) The Author(s). 2017 Open Access This article is distributed under the terms of the Creative Commons Attribution 4.0 International License (http://creativecommons.org/licenses/by/4.0/, which permits unrestricted use, distribution, and reproduction in any medium, provided you give appropriate credit to the original author(s) and the source, provide a link to the Creative Commons license, and indicate if changes were made. The Creative Commons Public Domain Dedication waiver (http://creativecommons.org/publicdomain/zero/1.0/) applies to the data made available in this article, unless otherwise stated. 


\section{Background}

\section{Dementia as a public health priority in older people}

Recent decades have seen rapid demographic ageing with numbers of people with dementia growing quickly worldwide $[1,2]$. Most recent regional estimates of the agestandardized prevalence of dementia in people aged 60 years and over fall between 5.6 and 7.6\%. Although dementia was traditionally viewed as more prevalent in developed countries, these estimates range from $4.6 \%$ in Central Europe to 8.7\% in North Africa and the Middle East [1].

Dementia has become a health and social care priority in many high-income countries, where, on the other hand, epidemiological evidence is becoming increasingly out of date [1]. Findings from some recent European studies $[3,4]$ have stimulated a renewed debate on secular trends for dementia prevalence [5-7]. It has been recommended that all countries should monitor trends regarding dementia prevalence and burden of disease, through nationally representative surveys to be regularly repeated [1, 2]. This particularly applies to Portugal, where epidemiological data regarding cognitive impairment and dementia are scarce.

\section{The situation in Portugal}

Portugal, a south European country, is still a step behind most developed western countries concerning some sociocultural indicators (as reflected by an illiteracy rate of $5.2 \%$ in 2011) [8]. The proportion of the Portuguese population aged 65 years and older has grown from $16.4 \%$ in 2001 to $19.0 \%$ in 2011 [8]. This age group still has a relatively low educational level (illiteracy rate of $19.5 \%$ in 2011) [8] and many will have lived under conditions of economic adversity and undernutrition in childhood, possibly increasing dementia risk.

Portuguese epidemiological studies in the field of cognitive impairment are easy to summarize. In the early 1990's, the EURODEM group were not able to include any studies from Portugal when reporting only trivial differences in the age-specific prevalence of dementia and Alzheimer's disease (AD) between participating European countries [9]. In 1994, Garcia et al. estimated the number of Portuguese people with dementia $(92,470)$ and AD $(48,706)$, based on European community prevalence rates [10]. These figures were updated by Eurocode to 153,000 and 90,000 [11], and more recently by Santana et al. [12] (in those of 60 years or over) to 160,287 and between 80,144-112,201, respectively.

Nunes et al. conducted the first Portuguese true epidemiological study of neurocognitive disorders, the POLSCI study [13]. In northern Portugal, they reported prevalences of $2.7 \%$ (CI 95\% 1.9-3.8\%) for DSM-IV dementia, with similar proportions of $\mathrm{AD}$ and vascular dementia subtypes, and $12.3 \%$ (CI95\% 10.4-14.4\%) for cognitive impairment 'no dementia'. However, the sample was not nationally representative. This and other methodological limitations preclude the generalization of POLSCI results to older populations in Portugal. On the other hand, the first national psychiatric epidemiological survey in Portugal unfortunately did not address dementia $[14,15]$.

\section{The 10/66 Dementia Research Group studies}

The 10/66 Dementia Research Group (10/66 DRG) was formed in 1998 to address the lack of dementia-related epidemiological research in low and middle income countries by: 1) devising a culture and education-fair diagnosis of dementia (the 10/66 DRG dementia diagnostic algorithm - henceforward referred to as '10/66 dementia'); 2) carrying out population-based surveys of the prevalence, incidence and impact of dementia in nine low and middle income countries (China, Cuba, Dominican Republic, India, Nigeria, Mexico, Peru, Puerto Rico, Venezuela) [16-19]. The 10/66 DRG protocols for prevalence and incidence surveys, together with a case-finder approach and a brief caregiver psychoeducational intervention, are detailed elsewhere [17]. The 10/66 DRG is committed to create an evidence base to empower advocacy, raise awareness about dementia, and ensure that the health and social care needs of older people are anticipated and met [17].

The implementation in Portugal of the 10/66 DRG protocols, beginning with the prevalence study, provided an opportunity to partially overcome the acknowledged insufficiencies of research in the field [20]. First, this is a fully structured approach, with a fixed diagnostic algorithm [17], lending itself to reassessments of prevalence over time, free of the bias of ever changing diagnostic practices. Second, the method provides comprehensive assessments of sociodemographic characteristics, mental disorders (including depression), physical health, anthropometry, non-communicable disease risk factors, disability/functioning, use of services, care arrangements and caregiver strain [17]. Third, there are concerns that the use of DSM-IV criteria may be systematically underestimating true prevalence in low education, low awareness settings (of which rural Portugal may still be an example). Finally, although the 10/66 DRG diagnostic algorithm has not yet been formally validated in a European context, there is robust evidence of its validity aside from the original validation study [21]: in Cuba (criterion validity against local specialist clinicians' diagnoses) [18]; in Chennai, India (predictive validity) [22]; in Lebanon (including in persons with mild to moderate dementia) [23]; and also in Singapore (a high income country) [24]. 


\section{Aims of the study}

We aimed to conduct a prevalence study of neuropsychiatric conditions in Portuguese older people, using the 10/66 DRG protocols. This was an opportunity to implement the validated 10/66 protocols for the first time in a European country, while estimating the prevalence of dementia. Data on depression, overall disability, caregiver strain and arrangements, along with service use, will be reported subsequently.

\section{Methods}

\section{Study design}

We applied the aforementioned 10/66 DRG guidelines for the dementia prevalence studies [17, 20]. A standardized operating procedures manual, covering every aspect of the training and field procedures, is available at http://www.alz.co.uk/1066/.

\section{Setting}

Two catchment areas were mapped, one in Fernão Ferro (Seixal, Setúbal), the other in Mora/Cabeção (Mora, Évora). Both locations are in the south of Portugal. Fernão Ferro, near Lisbon, is considered an urban setting, with predominately lower and middle class residents. Total population is 17,059 (675.3 inhabitants/ $\mathrm{km}^{2} ; 50.9 \%$ female; $18.9 \%$ above 64 years; $3.5 \%$ illiterate) [8, 25]. Mora/Cabeção, near Évora, includes a small town in the middle of a rural area. Total population is 3595 (21.2 inhabitants $/ \mathrm{km}^{2} ; 52.2 \%$ female; $32.4 \%$ above 64 years; $14.4 \%$ illiterate) $[8,25]$. In this paper, we will address the two catchment areas, mapped in each of the described locations, as 'urban (Fernão Ferro)' and 'rural (Mora)'. Mapping was carried out to identify each distinct household, prior to door-knocking to identify potential eligible participants (aged 65 years and over) with final eligibility confirmed during fieldwork.

\section{Participants}

In each catchment area, all residents aged 65 and over were approached by the research team. The only exclusion criteria were the inability to provide informed consent and the absence of a suitable representative for this purpose. For each participant, a reliable informant was appointed by consensus within each household. In most cases, this was the closest family member and a coresident, but the main criterion was to choose the person who knew the participant best (being or not a member of the household, or the family). Time spent with the older person was helpful in this decision. Where it was readily apparent that the older person needed care, the main caregiver was selected. Regarding the present study, we do not report on nursing home residents, part of which were also evaluated provided they had been living within the catchment area prior to admission.

\section{Measures}

The full array of measures and corresponding details may be found elsewhere [17, 20]. In short, 10/66 dementia and DSM-IV dementia diagnoses were established from: 1) the Geriatric Mental State (GMS), a semistructured clinical mental state interview which applies a computer algorithm (AGECAT), identifying organicity (probable dementia), depression, anxiety and psychosis [26]; 2) a cognitive test battery, including the Community Screening Instrument for Dementia (CSI'D') COGSCORE (which incorporates the CERAD animal naming verbal fluency task) and the modified CERAD 10 word list learning task with delayed recall (for each test, lower scores indicated more cognitive impairment); 3) a brief physical and neurological examination; 4) an informant interview including the structured CSI-D informant interview RELSCORE scale (summarising informant assessment of functional and cognitive decline - lower scores indicating less cognitive impairment) and the modified History and Aetiology Schedule - Dementia Diagnosis and Subtype (HAS-DDS) to establish the onset and course of dementia for subtype determination.

Information on the age and educational level of the participant, and household assets was obtained directly, or from the key informant where information was unreliable. The participant interview also included the World Health Organization Disability Assessment Schedule 2.0 (WHODAS 2.0) [27], and the informant interview included assessment of care arrangements, and the brief version of the Neuropsychiatric Inventory (NPI-Q) [28], assessing both severity of the symptoms and related caregiver distress.

Validated translations were already available for the NPI-Q [29]. All other assessments were carefully translated into European Portuguese, directly from the English originals. Discussions among Portuguese authors (AMC, JAS, MGP and MX) and cross-checking of backtranslations with $\mathrm{CF}$ and MP all helped to refine the translations. Pilot studies ensured the feasibility of these assessments and provided inputs into the final versions of Portuguese translations.

Dementia diagnosis was made using both 10/66 [17] and DSM-IV research criteria [30]. The corresponding algorithms have been validated and reported elsewhere $[18,31]$. Dementia subtypes were also assessed using an algorithm previously described [17], matching the NINDCS-ADRA Alzheimer's Disease [32], NINDSAIREN vascular dementia [33] and Lewy Body dementia [34] research diagnostic criteria. All the necessary information for applying this algorithm was retrieved from 10/66 DRG assessments (e.g. HAS-DDS), which also identify other prevalent conditions relevant to the differential diagnosis of dementia and dementia subtype (e.g. psychosis, depression, stroke). Severity of dementia, 
classified as questionable, mild, moderate or severe, was assessed according to the clinical dementia rating (CDR) [35]. This CDR summary score was calculated through an algorithm using 5-point scales on six domains of cognitive and functional performance (memory, orientation, judgment and problem solving, community affairs, home and hobbies, and personal care).

\section{Preparation and procedures}

There were two fieldwork operational supervisors (AMC, $\mathrm{CR}$ ) and 14 interviewers, distributed between the two areas. All the interviewers were mental health professionals (psychologists, nurses), acquainted with the study protocol and rigorously trained in the assessment procedures. Training, with focus on inter-rater reliability, was mainly conducted by two psychiatrists (MGP, MX) after attending a one-week meeting for 10/66 DRG researchers run by MP and CF at the Institute of Psychiatry/KCL. Cognitive and neurological/physical assessments were taught by a clinical neurologist (AV). A two-day workshop for the GMS was also conducted in Lisbon by MP, CF, MX and MGP.

Interviews were carried out directly in participant's own homes or scheduled to primary care or local community facilities, as convenient. All participants received the full assessment, which lasted on average 2-3 h. Sometimes, given the length of evaluation procedures, interviews were split (and assessments completed within no more than one week).

All data were collected directly onto laptop computers using computerized European Portuguese questionnaires driven by Epidata (version 3.1) software. These questionnaires had been developed by the 10/66 DRG, incorporating conditional skips and interactive checking of data consistency. Data were extracted into SPSS ${ }^{\circ}$ (version 21.0), and cleaning, processing of derived variables, and diagnostic algorithms were done with SPSS syntax files.

\section{Quality assurance}

From the starting of fieldwork, supervisors held monthly meetings with the local team of interviewers and supervised them randomly in the field. Quality control of collected data was assured by reviewing every completed interview, looking for missing data or inconsistencies. A random selection of interviews was thoroughly checked on a monthly basis by the study coordinators, to increase the accuracy of data entry. Data on households, participants and informants were stored in secure repositories, separated from data collected in interviews.

\section{Statistical analysis}

Participant characteristics were presented as frequencies and stratified by study site. Chi-square tests were conducted to assess any demographic differences between the two sites.
Using the same approach of Subramaniam and colleagues [24], the concurrent validity of dementia diagnoses with the DSM-IV and 10/66 criteria was first assessed by comparing the mean cognitive scores, WHODAS 2.0 disability scores, NPI severity and distress scores, and care needs as expressed by caregivers of respondents with no dementia (group 1), those with 10/66 dementia not confirmed by DSM-IV (group 2), and those with DSM-IV dementia (group 3). Differences among the groups were assessed using Chi-square tests for categorical variables, and Scheffe's test for multiple between group comparisons.

The prevalence of both DSM-IV and 10/66 dementia was estimated separately in urban and rural areas, and stratified by sex and age group. Among dementia cases the distribution of severity and subtype was described.

Finally, the effect of socio-demographic factors (age, sex, education level and number of assets) was modelled using a Poisson regression model, and presented using prevalence ratios.

Data were analysed using the Statistical Package for the Social Science for Windows 21.0 (IBM SPSS, Inc.) and STATA (StataCorp. 2011. Stata Statistical Software: Release 14. College Station, TX: StataCorp LP).

\section{Results}

\section{General description of the two samples}

We completed 1405 interviews with communitydwelling old age participants overall, 697 in the urban area (within Fernão Ferro) and 708 in the rural area (within Mora). In urban Fernão Ferro, 987 potential respondents had been identified in the defined catchment area, but 290 of these (or their representatives) refused to participate, could not be contacted after at least three attempts, or were unable to give informed consent. In rural Mora, 731 potential respondents had been identified but 23 (or their representatives) were not included in this study due to the same reasons. Therefore, participation rates were $81.8 \%$ overall, $70.6 \%$ in Fernão Ferro and $96.9 \%$ in Mora.

Urban participants tended to be younger, better educated, more likely to be married, and less likely to have worked as unskilled manual labourer or in agriculture than their rural counterparts (Table 1). There were no significant differences in gender between the two areas, whereas number of household assets tended to be higher in urban Fernão Ferro. Living alone was more frequent in rural Mora. Pension coverage was high in both regions, even if significantly lower in the urban sample $(86.1 \%$ vs $94.1 \%)$. Income from other sources (paid work, family transfers, rents, others) was uncommon, regardless of the area considered. Full dementia diagnoses could not be attributed to eight individuals 
Table 1 Social-demographic characteristics in the urban and rural areas

\begin{tabular}{|c|c|c|c|c|c|c|}
\hline & Total $(n=1405)$ & Urban $(n=697)$ & Rural $(n=708)$ & $x^{2}$ & $d f$ & $\mathrm{P}$ \\
\hline Age group (MV) & 0 & 0 & 0 & & & \\
\hline $65-69$ years & 359 & $193(27.7 \%)$ & $166(23.5 \%)$ & 28.0 & 3 & $<0.001$ \\
\hline $70-74$ years & 353 & 196(28.1\%) & $157(22.2 \%)$ & & & \\
\hline $75-79$ years & 340 & $175(25.1 \%)$ & 165(23.3\%) & & & \\
\hline $80+$ years & 353 & 133(19.1\%) & $220(31.1 \%)$ & & & \\
\hline Gender (MV) & 0 & 0 & 0 & & & \\
\hline Female & 779 & $373(53.5 \%)$ & $406(57.3 \%)$ & 2.1 & 1 & 0.149 \\
\hline Educational level (MV) & 70 & $57(8.2 \%)$ & $13(1.8 \%)$ & & & \\
\hline No education & 225 & $75(10.8 \%)$ & $150(21.2 \%)$ & 124.9 & 1 & $<0.001$ \\
\hline Primary (not completed) & 272 & $78(11.2 \%)$ & 194(27.4\%) & & & \\
\hline Completed primary & 770 & $446(64.0 \%)$ & $324(45.8 \%)$ & & & \\
\hline Completed secondary & 49 & $31(4.5 \%)$ & $18(2.5 \%)$ & & & \\
\hline Completed tertiary & 19 & $10(1.4 \%)$ & $9(1.3 \%)$ & & & \\
\hline Marital status (MV) & 7 & 1 & 6 & & & \\
\hline Never married & 61 & $19(2.7 \%)$ & $42(5.9 \%)$ & 35.9 & 4 & $<0.001$ \\
\hline Married or cohabiting & 946 & $509(73.0 \%)$ & $437(61.7 \%)$ & & & \\
\hline Widowed & 349 & $140(20.1 \%)$ & $209(29.5 \%)$ & & & \\
\hline Divorced or separated & 42 & $28(4.0 \%)$ & $14(2.0 \%)$ & & & \\
\hline Previous occupation (MV) & 24 & 27 & 40 & & & \\
\hline Professional/managerial & 171 & $127(18.2 \%)$ & $44(6.2 \%)$ & 88.2 & 4 & $<0.001$ \\
\hline Trade/clerical & 197 & $123(17.7 \%)$ & $74(10.4 \%)$ & & & \\
\hline Skilled laborer & 520 & $256(36.7 \%)$ & 264(37.3\%) & & & \\
\hline Unskilled manual laborer/agricultural worker & 497 & $185(26.5 \%)$ & $312(44.1 \%)$ & & & \\
\hline Living arrangements (MV) & 0 & 0 & 0 & & & \\
\hline Alone & 295 & $102(14.6 \%)$ & 193(27.3\%) & 57.6 & 4 & $<0.001$ \\
\hline With spouse only & 767 & $388(55.7 \%)$ & $379(53.5 \%)$ & & & \\
\hline With adult children & 30 & $25(3.6 \%)$ & $5(0.7 \%)$ & & & \\
\hline Other situations & 313 & $182(26.1 \%)$ & $131(18.1 \%)$ & & & \\
\hline Source of income (MV) & 0 & 0 & 0 & & & \\
\hline Any government or occupational pension & 1266 & $600(86.1 \%)$ & $666(94.1 \%)$ & 25.1 & 1 & $<0.001$ \\
\hline Number of household assets & 0 & 0 & 0 & & & \\
\hline $0-3$ assets & 5 & $2(0.3 \%)$ & $3(0.4 \%)$ & 15.1 & 1 & $<0.001$ \\
\hline $4-5$ assets & 57 & $14(2.0 \%)$ & $43(6.2 \%)$ & & & \\
\hline $6-7$ assets & 1343 & $681(97.7 \%)$ & $662(93.5 \%)$ & & & \\
\hline
\end{tabular}

due to missing information, hence creating an analysable sample size of 1397.

\section{Concurrent validation of $10 / 66$ and DSM-IV dementia diagnoses}

Results of the concurrent validation of 10/66 DRG and DSM-IV diagnoses are displayed in Table 2. Cognitive function (CSI-D COGSCORE) was significantly higher, and behavioural and psychological symptoms (NPI-Q), informant CSI-D RELSCORE and WHODAS 2.0 disability scores significantly lower in participants not diagnosed with dementia as compared to those with either DSM-IV dementia, or 10/66 dementia not confirmed by DSM-IV criteria. The distribution of these scores was more similar between those with DSM-IV dementia and those with 10/66 dementia not confirmed by DSM-IV, although the CSI-D RELSCORE and NPI-Q severity and carer distress scores were significantly higher in DSM-IV cases. Relevant needs for care were present 
Table 2 Concurrent validation of 10/66 and DSM-IV dementia diagnoses

\begin{tabular}{|c|c|c|c|c|c|c|c|}
\hline & & $\begin{array}{l}\text { Group 1: } \\
\text { No dementia } \\
(n=1268)\end{array}$ & $\begin{array}{l}\text { Group 2: 10/66 dementia } \\
\text { not confirmed by } \\
\text { DSM-IV }(n=105)\end{array}$ & $\begin{array}{l}\text { Group 3: } \\
\text { DSM-IV dementia } \\
(n=24)\end{array}$ & $\begin{array}{l}\text { Group } 1 \text { vs } \\
\text { Group } 2\end{array}$ & $\begin{array}{l}\text { Group } 1 \text { vs } \\
\text { Group } 3\end{array}$ & $\begin{array}{l}\text { Group } 2 \text { vs } \\
\text { Group } 3\end{array}$ \\
\hline \multirow[t]{2}{*}{ CSI'D COGSCORE } & Mean (SD) & $29.8(2.5)$ & $20.6(7.3)$ & $17.5(7.4)$ & \multirow{2}{*}{$\begin{array}{l}\text { t value }=-9.1 \\
p<0.001\end{array}$} & \multirow{2}{*}{$\begin{array}{l}\mathrm{t} \text { value }=-12.3 \\
p<0.001\end{array}$} & \multirow{2}{*}{$\begin{array}{l}\text { t value }=-3.1 \\
p=0.061\end{array}$} \\
\hline & Median (IQR) & $30.3(28.4-31.6)$ & $22.1(18.7-25.3)$ & $20.1(14.2-21.6)$ & & & \\
\hline \multirow[t]{2}{*}{ CSI'D RELSCORE } & Mean & $1.3(1.7)$ & $10.5(9.1)$ & $20.4(7.1)$ & \multirow{2}{*}{$\begin{array}{l}\text { t value }=9.2 \\
p<0.001\end{array}$} & \multirow{2}{*}{$\begin{array}{l}\text { t value }=19.1 \\
p<0.001\end{array}$} & \multirow{2}{*}{$\begin{array}{l}\mathrm{t} \text { value }=9.9 \\
p<0.001\end{array}$} \\
\hline & Median & $1(0-2)$ & $8(3-16)$ & $21.5(17-24.4)$ & & & \\
\hline \multirow[t]{2}{*}{ NPI-Q Distress } & Mean & $1.2(1.9)$ & $3.3(3.6)$ & $7.4(6.0)$ & \multirow{2}{*}{$\begin{array}{l}\mathrm{t} \text { value }=2.1 \\
p<0.001\end{array}$} & \multirow{2}{*}{$\begin{array}{l}\mathrm{t} \text { value }=6.2 \\
p<0.001\end{array}$} & \multirow{2}{*}{$\begin{array}{l}\mathrm{t} \text { value }=4.06 \\
p<0.001\end{array}$} \\
\hline & Median & $0(0-2)$ & $2(0-5)$ & $6(2-12)$ & & & \\
\hline \multirow[t]{2}{*}{ NPI-Q Severity } & Mean & $1.4(2.7)$ & $4.4(6.1)$ & $10.8(8.6)$ & \multirow{2}{*}{$\begin{array}{l}\mathrm{t} \text { value }=3.0 \\
p<0.001\end{array}$} & \multirow{2}{*}{$\begin{array}{l}\mathrm{t} \text { value }=9.3 \\
p<0.001\end{array}$} & \multirow{2}{*}{$\begin{array}{l}\mathrm{t} \text { value }=6.4 \\
p<0.001\end{array}$} \\
\hline & Median & $0(0-2)$ & $2(0-7)$ & $8(3-17)$ & & & \\
\hline \multirow{2}{*}{$\begin{array}{l}\text { WHODAS } 2.0 \\
\text { Disability }\end{array}$} & Mean & $16.9(19.2)$ & $41.1(30.0)$ & $52.8(31.9)$ & \multirow{2}{*}{$\begin{array}{l}\text { t value }=24.2 \\
p<0.001\end{array}$} & \multirow{2}{*}{$\begin{array}{l}\text { t value }=35.9 \\
p<0.001\end{array}$} & \multirow{2}{*}{$\begin{array}{l}\mathrm{t} \text { value }=11.7 \\
p=0.09\end{array}$} \\
\hline & Median & $11.1(0-27.8)$ & $37.5(15.3-66.7)$ & $55.5(27.8-80.56)$ & & & \\
\hline \multirow[t]{3}{*}{ Need for care } & $\begin{array}{l}\text { Needs care } \\
\text { much of the time }\end{array}$ & $2.1 \%$ & $33.3 \%$ & $54.2 \%$ & \multirow{3}{*}{\multicolumn{2}{|c|}{$X^{2}=381.7 p<0.0001$}} & \\
\hline & $\begin{array}{l}\text { Needs care } \\
\text { some of the time }\end{array}$ & $6.1 \%$ & $24.8 \%$ & $25.0 \%$ & & & \\
\hline & No care needed & $91.9 \%$ & $41.9 \%$ & $21.0 \%$ & & & \\
\hline
\end{tabular}

Respondents diagnosed with dementia by DSM-IV and also 10/66 diagnoses were defined as 'DSM-IV dementia' group ( $n=24)$. Respondents not diagnosed with dementia either by DSM-IV or 10/66 diagnoses were defined as 'No dementia' group $(n=1268)$. Respondents diagnosed with dementia by $10 / 66$ diagnosis but not confirmed by DSM-IV diagnosis were defined as '10/66 dementia not confirmed by DSM-IV' ( $n=105)$ group. CSI'D COGSCORE Community Screening Instrument for Dementia Global Cognitive Score, CSI'D RELSCORE Community Screening Instrument for Dementia Informant Score, NPI-Q Neuropsychiatric Inventory, WHODAS 2.0 World Health Organization Disability Assessment Schedule

both in persons with 10/66 and with DSM-IV dementia (58.1\% and $79.2 \%$, respectively, compared with $8.2 \%$ of those with no dementia). However, caregivers of persons with DSM-IV dementia reported that these participants needed care 'much of the time' more often (54.2\%) than those of persons with 10/66 dementia (33.3\%) (Table 2).

Prevalence of dementia, and distribution of subtypes and severity among dementia cases

The overall prevalence of dementia was 9.23 (95\% CI: 7.80-10.90) according to $10 / 66$ DRG criteria and 3.65 (95\% CI: 2.79-4.97) according to DSM-IV criteria (Table 3). The prevalence of 10/66 dementia was similar between the two sites, but in the urban site prevalence of DSM-IV dementia was almost double that in the rural site. Pure AD seemed to be the most prevalent subtype in both areas, especially in the urban area, although around one third of the cases could not be allocated to any particular subtype (Table 4).

CDR clinical severity was generally higher in persons with DSM-IV dementia as compared to those with 10/ 66 dementia. Questionable diagnoses of DSM-IV dementia were almost inexistent (i.e. one in 51), whereas questionable 10/66 dementia diagnoses represented around one fifth $(21.7 \%)$ of the total. However, even after excluding all questionable diagnoses, the 10/66 dementia prevalence rate surpassed that of DSM-IV dementia in both areas (Table 5).

\section{Correlates of dementia}

Table 6 presents data regarding some of the sociodemographic correlates of dementia. The prevalence of dementia was strongly age-dependent for both criteria, and in both sites (Table 3). There was a consistent trend for an inverse association between household assets and the prevalence of dementia. There were also either significant inverse associations between education level and dementia prevalence (urban 10/66 dementia) or trends in that direction (rural 10/66 dementia and urban DSM-IV). However, in the rural area higher education levels were associated with a higher prevalence of dementia. There were no significant associations with sex.

\section{Discussion}

We conducted a comprehensive one-phase survey of two catchment areas, one urban and the other rural, in the south of Portugal, with the main objective of estimating the prevalence of dementia. By adopting the 10/66 DRG protocols, we simultaneously diagnosed depression and other psychiatric disorders, which will be reported subsequently. We were interested in the crowded urban/suburban areas near Lisbon (the capital), as well as in the low population-density rural inner country, where risk factors for dementia may differ. One of these latter regions is Alentejo, with a high proportion of older people (24.2\%) and educational levels amongst the lowest in Europe (9.6\% illiterate) [25]. The choice of our urban and rural catchment areas reflected these 
Table 3 Prevalence of dementia in the two areas (urban and rural) and overall

\begin{tabular}{|c|c|c|c|c|c|c|}
\hline DSM-IV & & $65-69$ & $70-74$ & $75-79$ & $80+$ & Overall \\
\hline \multirow[t]{2}{*}{ Urban $(n=690)$} & Female & $\#$ & $6.93(3.33-13.80)$ & $4.60(1.73-11.63)$ & 11.11 (5.65-20.70) & $4.35(3.06-6.14)$ \\
\hline & Male & $1.18(0.17-7.90)$ & $2.15(0.54-8.21)$ & $5.88(2.47-13.38)$ & $5.08(1.65-14.64)$ & \\
\hline \multirow[t]{2}{*}{ Rural $(n=707)$} & Female & $\#$ & $1.05(0.15-7.11)$ & $1.16(0.16-7.82)$ & $6.06(3.10-11.63)$ & $2.97(1.95-4.49)$ \\
\hline & Male & $\#$ & $\#$ & $5.13(1.93-12.91)$ & 7.95 (3.83-15.79) & \\
\hline \multirow[t]{2}{*}{ Overall $(n=1397)$} & Female & $\#$ & $4.08(2.05-7.96)$ & $2.89(1.21-6.77)$ & $7.84(4.86-12.41)$ & 3.65 (2.79-4.97) \\
\hline & Male & $0.63(0.09-4.37)$ & $1.29(0.32-5.02)$ & $5.52(2.89-10.28)$ & $6.80(3.69-12.20)$ & \\
\hline $10 / 66$ & & $65-69$ & $70-74$ & $75-79$ & $80+$ & Overall \\
\hline \multirow[t]{2}{*}{ Urban $(n=690)$} & Female & $0.93(0.13-6.30)$ & $9.09(5.40-17.45)$ & $10.34(5.47-18.72)$ & $25.00(16.34-36.26)$ & $9.28(7.24-11.80)$ \\
\hline & Male & $2.35(0.59-8.94)$ & $3.23(1.04-9.55)$ & 11.76 (6.44-20.52) & $18.64(10.63-30.64)$ & \\
\hline \multirow[t]{2}{*}{ Rural $(n=707)$} & Female & $\#$ & $3.16(1.02-9.36)$ & $9.30(4.70-17.57)$ & $16.67(11.27-23.96)$ & $9.19(7.29-11.54)$ \\
\hline & Male & $2.74(0.70-10.15)$ & $9.68(4.47-19.72)$ & 10.26 (5.20-19.23) & 18.19 (11.43-27.69) & \\
\hline \multirow[t]{2}{*}{ Overall $(n=1397)$} & Female & $0.50(0.07-3.50)$ & $6.63(3.89-11.10)$ & $9.83(6.19-15.26)$ & $19.61(14.74-25.61)$ & $9.23(7.80-10.90)$ \\
\hline & Male & $2.53(0.96-6.52)$ & $5.81(3.06-10.75)$ & $11.04(7.06-16.86)$ & $18.37(12.89-25.49)$ & \\
\hline
\end{tabular}

\# no cases; $95 \%$ confidence intervals are presented into brackets

interests. In the total community sample, the prevalence of dementia was $9.23 \%$ (95\% CI: 7.80-10.90) using the 10/66 DRG algorithm and 3.65\% (95\% CI: 2.79-4.97) using DSM-IV criteria.

\section{Contextualisation}

The final sample size $(n=1405)$ was acceptable, as almost a third of Western European studies report sample sizes under 500 in this sort of studies [1]. In community epidemiological studies, response rates are usually somewhat higher in less developed settings, and those achieved in our study are consistent with, or somewhat better than other recent studies in high income countries [1]. Regarding comparisons between the urban and the rural area, the same research protocol was implemented in both by the same research group, which should warrant a regional comparison on solid grounds. However, potential bias cannot be excluded given higher participation rates in the rural area. In urban Fernão Ferro, a proportion of households turned

Table 4 10/66 dementia subtypes in the two areas (urban and rural) and overall

\begin{tabular}{llll}
\hline Dementia Subtype & \multicolumn{2}{l}{ AREA } \\
\cline { 2 - 4 } & $\begin{array}{l}\text { Urban } \\
\mathrm{n}(\%)\end{array}$ & $\begin{array}{l}\text { Rural } \\
\mathrm{n}(\%)\end{array}$ & $\begin{array}{l}\text { Overall } \\
\mathrm{n}(\%)\end{array}$ \\
\hline not allocated & $20(31.3)$ & $19(29.2)$ & $39(30.2)$ \\
pure Alzheimer's disease (AD) & $31(48.4)$ & $23(35.4)$ & $54(41.9)$ \\
pure vascular dementia (VAD) & $6(9.4)$ & $8(12.3)$ & $14(10.9)$ \\
mixed AD/ VAD & $0(0.0)$ & $7(10.8)$ & $7(5.4)$ \\
mixed AD/ Dementia with & $5(7.8)$ & $4(6.2)$ & $9(7.0)$ \\
Lewy Bodies (DLB) & & & \\
Frontal Temporal Dementia (FTD) & $2(2.9)$ & $4(6.2)$ & $6(4.7)$ \\
TOTAL & $64(100.0)$ & $65(100.0)$ & $129(100.0)$ \\
\hline
\end{tabular}

out to be weekend homes, and weaker social networks perhaps undermined the confidence of potential participants in allowing interviewers to their homes.

We found a higher prevalence of dementia using the 10/66 DRG diagnostic algorithm, compared to the prevalence of DSM-IV dementia, as seen previously across the other 10/66 sites [21]. While the prevalence of 10/66 dementia (9.2\%) was aligned within the range reported in other 10/66 DRG studies [21], the prevalence of DSM-IV dementia (3.7\%) might be seen as low in the light of recent reviews of the prevalence of dementia by world region [2]. The prevalence of DSM-IV dementia was lower, and the discrepancy between DSM-IV dementia and 10/66 dementia prevalence higher in the rural compared with the urban site. Just as in low and middle income countries, mild dementia may have been under-detected by DSM-IV criteria because of difficulties in establishing the criterion of social impairment, particularly in rural settings characterised by low education and limited awareness of dementia as a condition distinct from normal ageing [21].

Population prevalence may also have been underestimated since this was a study of the community prevalence, excluding formal residential care and nursing home settings. Community formal care is not well developed in Portugal, and most people with severe and advanced dementia would be expected to live in care homes. We did initially attempt to survey residential care and nursing homes located in our catchment areas. Unfortunately we were unable to obtain representative and unbiased estimates of prevalence in this stratum of the population because of problems gaining approval for access to some homes in the urban site that were operating informally without a full licence, and in gaining consent or assent for participation, particularly from those that did not have 
Table 5 Severity of dementia in the two areas (urban and rural) and overall

\begin{tabular}{|c|c|c|c|c|c|c|c|}
\hline & & DSM-IV der & & & 10/66 DRG dem & & \\
\hline & & Urban & Rural & Overall & Urban & Rural & Overall \\
\hline & Number of cases & 30 & 21 & 51 & 64 & 65 & 129 \\
\hline CDR & No dementia & 0 & 0 & 0 & $6(9.4 \%)$ & $2(3.1 \%)$ & $8(6.2 \%)$ \\
\hline & Questionable & 0 & $1(4.8 \%)$ & $1(2.0 \%)$ & $13(20.3 \%)$ & $15(23.1 \%)$ & $28(21.7 \%)$ \\
\hline & Mild & 19 (63.3\%) & $14(66.7 \%)$ & $33(64.7 \%)$ & $30(46.9 \%)$ & $36(55.4 \%)$ & $66(51.2 \%)$ \\
\hline & Moderate & $11(36.7 \%)$ & $6(28.6 \%)$ & 17 (33.3\%) & 15 (23.4\%) & $12(18.5 \%)$ & 27 (20.9\%) \\
\hline & $\begin{array}{l}\text { 10/66 dementia, restricted to } \\
\text { CDR mild/moderate/severe }\end{array}$ & & & & 7.53 (5.78-9.78) & $8.63(6.77-10.90)$ & 8.09 (6.77-9.64) \\
\hline
\end{tabular}

capacity to consent with no easily accessible nearest relative or legal representative. This may have had an important impact on the overall prevalence of dementia. In 2006 it was estimated that $3.4 \%$ of the Portuguese population aged 65 and over lived in care homes [36]; in the catchment areas we surveyed, our initial enumeration of facilities suggested that this proportion may approach $15 \%$, not all of whom would have met prior residence criteria for inclusion in our study.

As reported in many other studies we found that the prevalence of dementia increased with age, and was generally higher among those of lower socioeconomic status (as assessed through household assets). While the prevalence of dementia was also generally higher among those with less education, in the rural site only DSM-IV dementia prevalence but not 10/66 dementia prevalence was higher among those with more education. It is tempting to speculate that this counterintuitive finding may be explained by DSM-IV diagnostic bias operating selectively in low awareness settings.

\section{Comparison with European and Portuguese studies using other methods}

Five western European studies have recently reported a valid comparison of prevalence of dementia over time, but only two (in the UK and Spain) used the GMS as main tool [5], the same standardised psychiatric interview used here as part of the 10/66 DRG protocol. With a one-phase approach, Matthews et al. [3] found a prevalence rate of $6.5 \%$. This was significantly lower than the estimated rate of $8.3 \%$, challenging the idea of an 'epidemic-like' projection of dementia rates for the near future in Western countries. In Spain, Lobo et al. found a DSM-IV dementia prevalence of $3.9 \%$, in a two-phase study with case-reassessment by psychiatrists [4]. Regardless of methodological differences, this is close to the DSM-IV dementia prevalence in our study (3.7\%), possibly reflecting sociocultural similarities between Spanish and Portuguese populations.

Comparing with the POLSCI study in Northern Portugal [13], we found a slightly higher prevalence of DSM-IV dementia (3.65\%, 95\% CI: 2.79-4.97) vs 2.7\%, 95\% CI: 1.9-3.8). In POLSCI, the prevalence of dementia (DSM-IV criteria) was twice as high in the rural sample as compared with the urban one, with the rural/urban prevalence ratio increasing with age. In the present study, while we found that the prevalence of 10/66 dementia was similar in the urban and rural areas, DSM-IV dementia was almost double in the urban sample. There is no straightforward explanation for this contradiction in the results of the two studies, also given the fact that our urban sample tended to be younger and better educated, and the consistency of our findings with 10/66 DRG studies in other settings [21]. Perhaps the differences in response rates between rural and urban areas (which followed the same direction but were larger in our study) may account in part for these discrepancies. In POLSCI, $\mathrm{AD}$ and vascular dementia subtypes were similarly frequent among dementia cases, while in our study, although around $30 \%$ of cases could not be

Table 6 Prevalence ratios of socio-demographic factors associated within the two areas (urban and rural) and overall

\begin{tabular}{lllll}
\hline & & Urban & Rural & \multicolumn{1}{l}{ Overall } \\
\hline DSM-IV diagnosis of dementia & Age (per 5-year band) & $1.52(1.11-2.09)$ & $3.12(1.86-5.23)$ & $1.87(1.45-2.42)$ \\
& Male sex & $0.90(0.41-1.96)$ & $1.44(0.62-3.38)$ & $1.00(0.57-1.74)$ \\
& Education (per level) & $0.71(0.48-1.03)$ & $1.94(1.09-3.45)$ & $1.19(0.82-1.73)$ \\
& Assets (per asset) & $0.73(0.47-1.12)$ & $0.61(0.47-0.78)$ & $0.71(0.54-0.92)$ \\
$10 / 66$ DRG diagnosis of dementia & Age (per 5-year band) & $1.79(1.41-2.28)$ & $1.75(1.35-2.27)$ & $1.74(1.46-2.07)$ \\
& Male sex & $1.09(0.67-1.77)$ & $1.50(0.95-2.36)$ & $1.24(0.90-1.72)$ \\
& Education (per level) & $0.65(0.51-0.84)$ & $0.87(0.63-1.22)$ & $0.79(0.64-0.97)$ \\
& Assets (per asset) & $0.71(0.55-0.90)$ & $0.68(0.58-0.80)$ & $0.72(0.62-0.83)$ \\
\hline
\end{tabular}


allocated to any subtype, $\mathrm{AD}$ was more frequent than vascular dementia, particularly in the urban area.

Noteworthy, Nunes et al.'s results and ours are not directly comparable: both studies assessed nationally non-representative samples, in different regions of the Country and using different designs and assessments. In POLSCI there was a major interest in minor cognitive impairment, therefore the study did not fully address dementia prevalence and impact. For instance, sample age range (55-79 years) reflected a relatively young group, a limitation acknowledged by the authors [13]. Study design (a primary care-based two-phase survey lacking evaluation of a random sample of negative cases after screening), sample size $(n=1146)$ and overall participation rate (52.6\%) may have also influenced POLSCI results. Nevertheless, the overall impression is that prevalence rates for DSM-IV dementia were not strikingly different from those in our study, as confidence intervals widely overlap.

\section{Limitations and strength of the study}

First, by studying these two samples in different catchment areas, we obviously do not claim that the results are generalizable to the whole country. Despite its small territorial size, Portugal is not homogeneous regarding age distribution, literacy, income level, access to health services. However, given the common language and cultural background of the population, the implementation of the same protocol by the same team/supervisors favoured both the internal and external validity of our results.

Second, the criterion validity of 10/66 DRG dementia diagnoses was not established in Portugal by using a gold standard such as an examination by trained clinicians using standardized diagnosis criteria. However, this has been established in a variety of other settings [18, 24, 31], and the concurrent validation we carried out in this study provides further evidence to support construct validity in our context. Analysing predictive validity in our sample would be a complementary approach $[19,22]$, if feasible in the near future. Third, our inability to access and survey representative samples of older residents of residential care and nursing homes is a significant limitation. We were not able to accurately define the number of older people who could not be approached in institutions. Therefore we did not perform a sensitivity analysis to estimate the number of dementia cases and calculate an adjusted total prevalence in nursing home residents. Hopefully, much can be learnt from our experience, in designing a robust sampling methodology to correct this evidence gap in future national or regional research.

Ours is the first comprehensive one-phase survey of the community prevalence of dementia in Portuguese populations aged 65 and over. By accomplishing this first
10/66 DRG survey in a European country we showed that 10/66 DRG protocols and fieldwork procedures were feasible in this setting. They may be more appropriate for low education European populations such as these Portuguese ones, particularly in rural Mora where nearly half of the older participants had not completed primary education, and where the full validity of standard non-education fair cognitive assessments would be doubtful.

\section{Implications}

Old people with neuropsychiatric problems including dementia and depression represent a frail and important subgroup of the ageing population, whose needs, together with those of their caregivers, are still poorly understood and met. This is especially so in Portugal, were community prevalence studies are crucial, following the priorities set by the National Mental Health Plan 2007-2016 [37]. Only reliable and cross-culturally comparable information about the epidemiology and patterns of care of these conditions may guide adequate disease management [31]: as recently pointed out [7], using a fixed methodology has potential benefits to estimate changes in dementia prevalence [3] or incidence [38] in defined populations, and to foster international comparisons [1].

Regarding this endeavour, the 10/66 DRG protocols for prevalence studies provide robust opportunities as they have now been applied in Europe: 1) they tackle the main neuropsychiatric conditions in old age, dementia and depression; 2) the 10/66 one-stage approach has proven advantages [17] over the two-phase procedures used in most studies on dementia (i.e. screening followed by a diagnostic stage): attrition is marked between the first and other phases; participants with probable dementia are particularly likely to refuse, to move away or to die, leading to informative censoring [39, 40]; 3) catchment area surveys are logistically straightforward and have been favoured by dementia researchers.

\section{Conclusions}

For the first time the 10/66 DRG protocols were successfully implemented in Europe. As in other studies using the same protocol, we report a significant disparity between DSM-IV and 10/66 diagnoses, reinforcing the need to discuss diagnostic algorithms for dementia. Despite need for caution concerning interpretation, the prevalence rate of 10/66 dementia (9.2\%) suggests that previous studies possibly underestimated the true dementia prevalence rates in Portugal. Not least, estimates of numbers of Portuguese persons with dementia yielded figures [10-12] that would be clearly exceeded if our findings were nationally generalizable. 
Given its ageing population, Portugal is probably about to face a highly demanding health challenge in the coming years, as most of the national health budget is still allocated to hospital acute care instead of directed to chronic disease management. Our results additionally support the need for a new wave of dementia surveys in developed countries.

\begin{abstract}
Abbreviations
POLSCI: A Portuguese population-based longitudinal study (cf. reference 13); 10/66 DRG: Dementia Research Group; AD: Alzheimer's disease; AGECAT: The Automated Geriatric Examination for Computer Assisted Taxonomy; CDR: Clinical Dementia Rating; CERAD: Consortium to Establish a Registry for Alzheimer's Disease: Cl: Confidence intervals; CSI'D' COGSCORE: Community Screening Instrument for Dementia, cognitive score; CSI'D' RELSCORE: Community Screening Instrument for Dementia, informant score; DSM-IV: Diagnostic and Statistical Manual of Mental Disorders, fourth edition; EURODEM: European Community Concerted Action on Epidemiology and Prevention of Dementia; GMS: Geriatric Mental State examination; HAS-DDS: History and Aetiology Schedule - Dementia Diagnosis and Subtype; NINDCS-ADRA: National Institute of Neurological and Communicative Disorders and Stroke and the Alzheimer Disease and Related Disorders Association Criteria for Alzheimer Disease; NINDS-AIREN vascular dementia: National Institute of Neurological Disorders and Stroke and Association Internationale pour la Recherche et I'Enseignement en Neurosciences; NPI-Q: Neuropsychiatric Inventory - Questionnaire, brief version; WHODAS 2.0: World Health Organization Disability Assessment Schedule version 2.0
\end{abstract}

\section{Acknowledgements}

The following research assistants contributed to data collection: A. Viegas, C. Simões, F. Barreiros, I. Magalhães, P. L. Dias and R. Campaniço (Fernão Ferro); P. Gens, F. Costa, M. José Vicente, M. Calhau, R. Bairrão Carvalho, S. Rosado, V. Gens and V. Mendes (Mora). The authors wish to thank to all local facilitators: USF Fernão Ferro mais, ARPIF (Associação de Reformados, Pensionistas e Idosos de Fernão Ferro), Junta de Freguesia de Fernão Ferro, Paróquia de Fernão Ferro, Santa Casa da Misericórdia de Mora, Mora Health Centre, Guarda Nacional Republicana and local pharmacies.

\section{Funding}

This study was supported by a research grant from FCT - Fundação para a Ciência e a Tecnologia, the Portuguese national funding agency for science, research and technology (Project reference PTDC/SAU-EPI/113652/2009: "Prevalence of old age neuropsychiatric disorders: contribution to mental health policy in Portugal"; PI: Miguel Xavier). A. Matthew Prina was supported by the MRC (MR/K021907/1). iNOVA4Health - UID/Multi/04462/2013, a program financially supported by Fundação para a Ciência e Tecnologia / Ministério da Educação e Ciência, through national funds and co-funded by FEDER under the PT2020 Partnership Agreement is acknowledged.

\section{Availability of data and materials}

The data that support the findings of this study are available from Nova Medical School/FCM-UNL but restrictions apply to the availability of these data, which were used under license for the current research, and therefore are not publicly available according to the Portuguese law. Data are however available from the authors upon reasonable request and with permission of the Nova Medical School/FCM-UNL Ethics Committee.

\section{Authors' contributions}

MGP, MX, CPF and MP applied the 10/66 DRG prevalence study protocols to the Portuguese context. AV, JAS, MCA, AF, AMC and CR collaborated in the design, implementation and monitoring of the study. AMC and CR were fieldwork operational supervisors, with substantial help from MCA and AF. MGP and MX coordinated fieldwork and quality assurance procedures. MGP, MX, AMP and MP drafted the initial manuscript. AMP was responsible for statistical analyses, together with MP. All authors contributed significantly to several critical revisions of the manuscript, and have read and approved its final version.

\section{Ethics approval and consent to participate}

Ethical approval was obtained from the Nova Medical School/FCM-UNL Ethics Committee (reference number 04/2012/CEFCM). Participants gave their written informed consent prior to assessments. In case they were unable to provide it, written informed consent was taken from their representative/next of kin.

\section{Consent for publication}

Not applicable.

\section{Competing interests}

The authors declare that they have no competing interests.

\section{Publisher's Note}

Springer Nature remains neutral with regard to jurisdictional claims in published maps and institutional affiliations.

\section{Author details \\ ${ }^{1}$ Chronic Diseases Research Center (CEDOC), Nova Medical School | Faculdade de Ciências Médicas, Universidade Nova de Lisboa, Campo Mártires da Pátria 130, 1169-056 Lisbon, Portugal. ${ }^{2}$ Department of Neurosciences, H. Santa Maria/CHLN; IMM and ISAMB, Faculty of Medicine, University of Lisbon, Lisbon, Portugal. ${ }^{3}$ Champalimaud Neuroscience Programme, Champalimaud Centre for the Unknown, Lisbon, Portugal. ${ }^{4}$ Santa Casa da Misericórdia de Mora, União das Misericórdias Portuguesas, Mora, Portugal. ${ }^{5}$ USF Fernão Ferro mais, ACES Almada-Seixal, Seixal, Portugal. ${ }^{6}$ King's College London, Centre for Global Mental Health, Health Service and Population Research, Institute of Psychiatry, Psychology \& Neuroscience, De Crespigny Park, London SE5 8AF, UK. `Universidade Federal de São Paulo, Department of Psychobiology, São Paulo, Brasil.}

Received: 23 March 2017 Accepted: 19 October 2017

Published online: 07 November 2017

\section{References}

1. Prince M, Wimo A, Guerchet M, Gemma-Claire A, Wu Y-T, Prina M, et al. World Alzheimer report 2015: the global impact of dementia - an analysis of prevalence, incidence, cost and trends. London: Alzheimer's Disease International (ADI); 2015.

2. Prince $M$, Bryce $R$, Albanese E, Wimo A, Ribeiro W, Ferri CP. The global prevalence of dementia: a systematic review and metaanalysis. Alzheimers Dement, Elsevier Ltd; 2013;9(1):63-75.e2.

3. Matthews FE, Arthur A, Barnes LE, Bond J, Jagger C, Robinson L, et al. A two-decade comparison of prevalence of dementia in individuals aged 65 years and older from three geographical areas of England: results of the Cognitive Function and Ageing Study I and II. Lancet. 2013;382:1405-12.

4. Lobo A, Saz P, Marcos G, Dia JL, De-la-Camara C, Ventura T, et al. Prevalence of dementia in a southern European population in two different time periods: the ZARADEMP project. Acta Psychiatr Scand. 2007;116:299-307.

5. Wu Y-T, Fratiglioni L, Matthews FE, Lobo A, Breteler MMB, Skoog I, et al. Dementia in western Europe: epidemiological evidence and implications for policy making. Lancet Neurol, Elsevier Ltd. 2016;15(1):116-24.

6. Langa KM. Is the risk of Alzheimer's disease and dementia declining? Alzheimers Res Ther. 2015;7:34.

7. Prince M, Ali G-C, Guerchet M, Prina AM, Albanese E, Wu Y-T. Recent global trends in the prevalence and incidence of dementia, and survival with dementia. Alzheimers Res Ther BioMed Central. 2016;8(1):23.

8. INE - Instituto Nacional de Estatística. Censos 2011: Resultados definitivos Portugal. Instituto Nacional de Estatística IP, Av. António José de Almeida, 1000-043 Lisboa, Portugal, editors. 2012.

9. Hofman A, Rocca WA, Brayne C, Breteler MMB, Clarke M, Cooper B, et al. The prevalence of dementia in Europe: a collaborative study of 1980-1990 findings. Int J Epidemiol. 1991;20:736-48.

10. Garcia C, Costa C, Guerreiro M, Leitão O, Mendonça A, Umbelino J. Estimativa da prevalência da demência e da doença de Alzheimer em Portugal [An estimate of the prevalence of dementia and Alzheimer's disease in Portugal]. Acta Medica Port. 1994;7(9):487-91.

11. Alzheimer Europe. European Collaboration on Dementia (EuroCoDe) technical report (2006-2008). Luxembourg: Alzheimer Europe; 2008.

12. Santana I, Farinha F, Freitas S, Rodrigues V, Carvalho Á. Epidemiologia da Demência e da Doença de Alzheimer em Portugal: Estimativas da Prevalência e dos Encargos Financeiros com a Medicação [The Epidemiology of Dementia and Alzheimer Disease in Portugal: Estimations of Prevalence and Treatment-Costs]. Acta Medica Port. 2015;28(2):182-8. 
13. Nunes B, Silva RD, Cruz VT, Roriz JM, Pais J, Silva MC. Prevalence and pattern of cognitive impairment in rural and urban populations from northern Portugal. BMC Neurol. 2010;10:42.

14. Xavier M, Baptista H, Mendes JM, Magalhães P, Caldas-de-Almeida JM. Implementing the World Mental Health Survey Initiative in Portugal - rationale, design and fieldwork procedures. Int J Ment Health Syst. 2013;7:19.

15. Caldas-de-Almeida JM, Xavier M, Cardoso G, Gonçalves-Pereira M, Gusmão RM, Corrêa BB, et al. Estudo Epidemiológico Nacional de Saúde Mental: $1^{\circ}$ relatório. Lisbon: Nova Medical School/Faculdade de Ciências Médicas Universidade Nova de Lisboa; 2013.

16. Prince M. Dementia in developing countries: a consensus statement from the 10/66 Dementia Research Group. Int J Geriatr Psychiatry. 2000;15:14-20.

17. Prince M, Ferri CP, Acosta D, Albanese E, Arizaga R, Dewey M, et al. The protocols for the 10/66 Dementia Research Group population-based research programme. BMC Public Health. 2007;7:165

18. Prince MJ, de Rodriguez JL, Noriega L, Lopez A, Acosta D, Albanese E, et al. The 10/66 Dementia Research Group's fully operationalised DSM-IV dementia computerized diagnostic algorithm, compared with the 10/66 dementia algorithm and a clinician diagnosis: a population validation study. BMC Public Health. 2008;8:219.

19. Prina AM, Acosta D, Acostas I, Guerra M, Huang Y, Jotheeswaran AT, et al. Cohort profile: the 10/66 study. Int J Epidemiol. 2017;46(2):406-406i.

20. Gonçalves-Pereira M, Cardoso A, Verdelho A, Alves da Silva J, Caldas de Almeida M, Fernandes A, et al. Implementação em Portugal de um estudo de prevalência da demência e da depressão geriátrica: a metodologia do 10/66 Dementia Research Group [Implementing a prevalence study of dementia and geriatric depression in Portugal: The 10/66 DRG methodology]. Rev Port. Saúde Pública, Elsevier. 2016;34:134-143.

21. Rodriguez JJL, Ferri CP, Acosta D, Guerra M, Huang Y, Jacob KS, et al. Prevalence of dementia in Latin America, India, and China: a populationbased cross-sectional survey. Lancet. 2008;372:464-74.

22. Jotheeswaran AT, Williams JD, Prince MJ. The predictive validity of the 10/66 dementia diagnosis in Chennai, India: a 3-year follow-up study of cases identified at baseline. Alzheimer Dis Assoc Disord. 2010;24(3):296-302.

23. Phung KTT, Chaaya M, Waldemar G, Atweh S, Asmar K, Ghusn H, et al. Validation of the 10/66 Dementia Research Group diagnostic assessment for dementia in Arabic: a study in Lebanon. J Geriatr Psychiatry Neurol. 2014; 27(4):282-90.

24. Subramaniam M, Chong SA, Vaingankar JA, Abdin E, Chua BY, Chua HC, et al. Prevalence of dementia in people aged 60 years and above: results from the WiSE study. J Alzheimers Dis. 2015;45(4):1127-38.

25. INE - Instituto Nacional de Estatística. CENSOS 2011 - Instituto Nacional de Estatística / CAOP 2013 - Carta Administrativa Oficial Portuguesa [Internet] 2013 [cited 2015 Jul 13]. Available from: http://censos.ine.pt

26. Copeland JR, Dewey ME, Griffiths-Jones HM. A computerized psychiatric diagnostic system and case nomenclature for elderly subjects: GMS and AGECAT. Psychol Med. 1986;16:89-99.

27. Rehm J, Üstün TB, Saxena S, Nelson CB, Chatterji S, Ivis F, et al. On the development and psychometric testing of the WHO screening instrument to assess disablement in the general population. Int J Methods Psychiatr Res. 1999:8:110-22

28. Kaufer DI, Cummings JL, Ketchel P, Smith V, MacMillan A, Shelley T, et al. Validation of the NPI-Q, a brief clinical form of the Neuropsychiatric Inventory. J Neuropsychiatr Clin Neurosci. 2000;12:233-9.

29. GEECD (Grupo de Estudos de Envelhecimento Cerebral e Demência). Escalas e Testes na Demência. 2nd ed; 2008.

30. APA (American Psychiatric Association). Diagnostic and Statistical Manual of Mental Disorders. 4th ed. Washington DC: APA; 1994.

31. Prince $M$, Acosta $D$, Chiu H, Scazufca M, Varghese M. Dementia diagnosis in developing countries: a cross- cultural validation study. Lancet. 2003;361:909-17.

32. McKhann G, Drachman D, Folstein M, Katzman R, Price D, Stadlan EM. Clinical diagnosis of Alzheimer's disease: report of the NINCDS-ADRDA work group under the auspices of Department of Health and Human Services Task Force on Alzheimer's disease. Neurology. 1984;34:939-44.

33. Román GC, Tatemichi TK, Erkinjuntti T, Cummings JL, Masdeu JC, Garcia JH, et al. Vascular dementia: diagnostic criteria for research studies. Report of the NINDS-AIREN international workshop. Neurology. 1993;43:250-60.

34. McKeith IG, Perry RH, Fairbairn AF, Jabeen S, Perry EK. Operational criteria for senile dementia of Lewy body type (SDLT). Psychol Med. 1992;22:911-22.
35. Morris JC. The clinical dementia rating (CDR): current version and scoring rules. Neurology. 1993;43:2412-4.

36. Joël ME, Dufour-Kippelen S, Samitca S. The long-term care system for the elderly in Portugal. European Network of Economic Policy Research Institutes (ENEPRI) Research report $n^{\circ} 84.2010$

37. CNRSSM-Comissão Nacional de Reestruturação dos Serviços de Saúde Mental. Relatório: Proposta de Plano de Acção para a Reestruturação e Desenvolvimento dos Serviços de Saúde Mental em Portugal 20072016. 2008.

38. Matthews FE, Stephan BCM, Robinson L, Jagger C, Barnes LE, Arthur A, et al. A two decade dementia incidence comparison from the Cognitive Function and Ageing Studies I and II. Nat. Commun. Nat Publ Group. 2016;7:11398.

39. Dunn G, Pickles A, Tansella M, Vázquez-Barquero JL. Two-phase epidemiological surveys in psychiatric research: editorial. Br J Psychiatry. 1999:174:95-100.

40. Prince M. Commentary: two-phase surveys. A death is announced; no flowers please. Int J Epidemiol. 2003;32:1078-80

\section{Submit your next manuscript to BioMed Central and we will help you at every step:}

- We accept pre-submission inquiries

- Our selector tool helps you to find the most relevant journal

- We provide round the clock customer support

- Convenient online submission

- Thorough peer review

- Inclusion in PubMed and all major indexing services

- Maximum visibility for your research

Submit your manuscript at www.biomedcentral.com/submit
Biomed Central 